TRANSACTIONS OF THE

AMERICAN MATHEMATICAL SOCIETY

Volume 362, Number 3, March 2010, Pages 1659-1680

S 0002-9947(09)04841-7

Article electronically published on October 20, 2009

\title{
BAIRE CLASSES OF BANACH SPACES AND STRONGLY AFFINE FUNCTIONS
}

\author{
JIŘí SPURNÝ
}

\begin{abstract}
We construct a metrizable simplex $X$ and a Baire-two function $f$ on $X$ satisfying the barycentric formula such that $f$ is not of affine class two; i.e., there is no bounded sequence of affine Baire-one functions on $X$ converging to $f$. This provides an example of a Banach $\mathcal{L}_{\infty}$-space $E$ such that $E_{2}^{* *} \neq E_{\mathcal{B}_{2}}^{* *}$.
\end{abstract}

\section{INTRODUCTION}

If $X$ is a compact space, let $\mathcal{C}(X)$ stand for the space of all continuous functions on $X, \mathcal{B}_{\alpha}(X)$ denote the space of all functions of Baire class $\alpha$ on $X, \mathcal{B}_{\omega_{1}}(X)=$ $\bigcup_{\alpha<\omega_{1}} \mathcal{B}_{\alpha}(X)$ stand for the space of all Baire functions and $\mathcal{B}(X)$ be the space of all Borel functions on $X$. We recall that a bounded function $f$ on $X$ is universally measurable if $f$ is measurable with respect to every Radon measure $\mu$ on $X$. We denote the space of all universally measurable functions $\mathcal{U}(X)$.

Let $\mathfrak{A}(X)$ stand for the space of all affine continuous functions on a compact convex subset $X$ of a locally convex space and let $\mathfrak{A}_{1}(X)$ denote the space of all functions of affine class one, i.e, the space of all pointwise limits of bounded sequences of affine continuous functions.

Inductively, for a countable ordinal number $\alpha$ we define the space $\mathfrak{A}_{\alpha}(X)$ of functions of affine class $\alpha$ as the family of pointwise limits of bounded sequences $\left\{f_{n}\right\}$, where functions $f_{n}$ are of lower affine classes (cf. [8]).

A function $f \in \mathcal{U}(X)$ is said to satisfy the barycentric formula if $\mu(f)=f(r(\mu))$ for any probability Radon measure $\mu$ on $X$. (Here $r(\mu)$ stands for the barycenter of $\mu$; we refer the reader to the next section for notions not explained here.) Functions satisfying the barycentric formula are sometimes termed strongly affine (see 26. Introduction]). It is easy to see that any strongly affine function is bounded (see [15, Satz 2.1]).

Let $\mathfrak{A}_{\mathrm{bf}}(\mathrm{X})$ stand for the space of all strongly affine functions on $X$. By a result of G. Choquet (see [1, Theorem I.2.6]), any affine Baire-one function $f$ on $X$ is bounded and strongly affine. By a result of G. Mokobodzki, $f$ is a pointwise limit of a bounded sequence of affine continuous functions on $X$ (see [22, Théorème 80]).

Received by the editors May 24, 2007 and, in revised form, June 4, 2008.

2000 Mathematics Subject Classification. Primary 46A55; Secondary 26A21.

Key words and phrases. Simplex, strongly affine functions, barycentric formula, Baire functions, $L^{1}$-preduals, intrinsic Baire classes.

This research was supported in part by the grants GA ČR 201/06/0018, GA ČR 201/07/0388, and in part by the Research Project MSM 0021620839 from the Czech Ministry of Education. 
In the terminology of M. Capon introduced above, affine Baire-one functions are of affine class one.

The question of a possible generalization of this result for affine functions of higher Baire classes was solved by M. Talagrand in [26, Theorem]. He constructed a strongly affine Baire-two function $f$ on a compact convex set $X$ such that $f \notin$ $\mathfrak{A}_{\alpha}(X)$ for every countable ordinal $\alpha$. The set $X$ also has a remarkable feature that any affine Baire-one function on $X$ is continuous and hence $\mathfrak{A}(X)=\mathfrak{A}_{\alpha}(X)$ for each $\alpha<\omega_{1}$.

If we turn our attention to simplices, the structure of spaces $\mathfrak{A}_{\alpha}(X)$ for a metrizable simplex $X$ is much richer in general. If $X$ is a metrizable simplex, whose set ext $X$ of all extreme points of $X$ is not closed, then there exists a continuous convex function $f$ on $X$ such that its upper envelope $f^{*}$ is not continuous (see [1, Theorem II.4.1]). Since $f^{*}$ is upper semicontinuous and $X$ is metrizable, $f^{*}$ is a noncontinuous affine Baire-one function on $X$ (see [1, Theorem II.3.7]).

If the set ext $X$ is countable, any strongly affine function $f$ on $X$ is of affine class 1. Indeed, the restriction of $f$ to ext $X$ is Baire-one, and hence there exists an extension $h \in \mathfrak{A}_{1}(X)$ satisfying $h=f$ on ext $X$ (see [8, Lemma 1.1]). Since $f$ is strongly affine, $f=h$ is of affine class 1 .

If the set ext $X$ is uncountable, then, as a $G_{\delta}$-subset of a compact metrizable set $X$ (see [1, Corollary I.4.4]), it contains a nonempty perfect set $C$. Since any bounded Baire function of class $\alpha$ on $C$ can be extended to a function of affine class $\alpha$ (this follows easily from [16, Theorem 3.6]; see e.g. [25, Remark 3.4]), $\mathfrak{A}_{\alpha}(X)$ is a proper subspace of $\mathfrak{A}_{\alpha+1}(X)$ for each $\alpha<\omega_{1}$.

There is a close relationship between strongly affine Baire- $\alpha$ functions and functions of affine class $\alpha$ on simplices; precisely, M. Capon showed in [8, Théorème 2] that any strongly affine Baire $-\alpha$ function on a simplex is of affine class $\alpha+1$.

The aim of our paper is to show that the shift of classes in the theorem of M. Capon can really occur. Precisely, we get the following result.

Theorem 1.1. There exists a metrizable simplex $X$ such that $\mathfrak{A}_{2}(X) \neq \mathcal{B}_{2}(X) \cap$ $\mathfrak{A}_{\mathrm{bf}}(\mathrm{X})$.

The Banach space $\mathfrak{A}(X)$ on a simplex $X$ is a particular case of an $L_{1}$-predual; i.e., the dual space $(\mathfrak{A}(X))^{*}$ is isometric to $L_{1}(\mu)$ for a suitable measure $\mu$ (see [11, Proposition 3.23]). According to [13, page 59], $\mathfrak{A}(X)$ is an $\mathcal{L}_{\infty, 1+\varepsilon^{-}}$space for each $\varepsilon>0$.

If $X$ is a Banach space, following 2, page 1044] we define intrinsic $\alpha$-Baire classes of the double dual $X^{* *}$ as follows. We set $X_{0}^{* *}=X \subset X^{* *}$ and for $\alpha \in$ $\left(0, \omega_{1}\right)$, let $X_{\alpha}^{* *}$ be the space of all weak* limits of sequences from $\bigcup_{\beta<\alpha} X_{\beta}^{* *}$.

If $X$ is considered to be a subspace of the space $\mathcal{C}(K)$, where $K$ is the dual unit ball of $X$ endowed with the weak* ${ }^{*}$-topology, the $\alpha$-th Baire class of $X^{* *}$ is defined as

$$
X_{\mathcal{B}_{\alpha}}^{* *}=\left\{x^{* *} \in X^{\perp \perp}:\left.x^{* *}\right|_{K} \in \mathcal{B}_{\alpha}(K)\right\} .
$$

We can also use the identification

$$
X_{\mathcal{B}_{\alpha}}^{* *}=\mathcal{B}_{\alpha}(K) \cap \mathfrak{A}_{\mathrm{bf}}(\mathrm{K}) \cap \mathfrak{A}_{\text {odd }}(\mathrm{K}),
$$

where $\mathfrak{A}_{\text {odd }}(K)$ stands for the space of all odd affine functions on $K$ ( $f$ is odd on $K$ if $\left.f\left(-x^{*}\right)=-f\left(x^{*}\right), x^{*} \in K\right)$. (We refer the reader to the survey paper [2, pages 1043-1048] for a detailed exposition of Baire classes of Banach spaces.) 
Obviously, $X_{\alpha}^{* *} \subset X_{\mathcal{B}_{\alpha}}^{* *}$ and $X_{1}^{* *}=X_{\mathcal{B}_{1}}^{* *}$ by the aforementioned result of G. Choquet and G. Mokobodzki (see also [2, Theorem II.1.2(a)]). If $\alpha \geq 2$, the equality need not hold anymore, as is witnessed by the aforementioned example of M. Talagrand.

It is claimed in [2, page 1047] that

$$
X_{\alpha}^{* *}=X_{\mathcal{B}_{\alpha}}^{* *}, \quad \alpha \in\left[0, \omega_{1}\right),
$$

provided $X$ is an $\mathcal{L}_{\infty}$-space. Nevertheless, in our opinion the validity of the proof presented there relies upon the weak ${ }^{*}$-continuity of the projection $Q$, which is not always satisfied. The space $\mathfrak{A}(X)$ constructed in Theorem 1.1 provides a counterexample to this statement.

Theorem 1.2. There exists a Banach space $X$ that is an $\mathcal{L}_{\infty, 1+\varepsilon}$-space for every $\varepsilon>0$ and $X_{2}^{* *} \neq X_{\mathcal{B}_{2}}^{* *}$.

It is not difficult to prove that $X_{\alpha}^{* *}=X_{\mathcal{B}_{\alpha}}^{* *}$ provided the space $X$ is complemented in some $\mathcal{C}(K)$-space (see Proposition 4.2). Thus, the constructed space $\mathfrak{A}(X)$ is an example of an $L_{1}$-predual that is not complemented in any $\mathcal{C}(K)$-space. The first space of this type was constructed in [7.

It might be interesting to remark that complementability of a Banach space $X$ in some $\mathcal{C}(K)$-space is a sufficient, but not necessary, condition for (1) to hold true.

Example 1.3. There exists a simplex $X$ such that $\mathfrak{A}(X)$ is not complemented in any $\mathcal{C}(K)$-space and $(\mathfrak{A}(X))_{\alpha}^{* *}=(\mathfrak{A}(X))_{\mathcal{B}_{\alpha}}^{* *}$ for every $\alpha \in\left[0, \omega_{1}\right)$.

The paper is organized as follows. Since the proof of Theorem 1.1 depends on the notion of a function space, the next section provides a general overview of function spaces and their properties relevant to our example. The third section of the paper is devoted to several topological results that will be substantially used in the main part. Auxiliary results on Baire classes of Banach spaces are presented in Section 4 . A construction of a suitable function space, which gives rise to the sought simplex, is presented in Section 5 and its properties are described in Section 6. All the ideas are put together in the final part.

\section{Preliminaries}

All topological spaces will be considered as Hausdorff. If $K$ is a compact space, we denote by $\mathcal{C}(K)$ the space of all continuous functions on $K$. We will identify the dual of $\mathcal{C}(K)$ with the space $\mathcal{M}(K)$ of all Radon measures on $K$. Let $\mathcal{M}^{1}(K)$ denote the set of all probability Radon measures on $K$ and let $\varepsilon_{x}$ stand for the Dirac measure at $x \in K$. If $\mathcal{H}$ is a subset of $\mathcal{C}(K)$, we write $\mathcal{H}^{\perp}$ for the space of all Radon measures $\mu$ on $K$ satisfying $\mu(h)=0$ for any $h \in \mathcal{H}$.

If $f$ is a bounded universally measurable function on $K$, we can regard it as an element of $(\mathcal{C}(K))^{* *}$ via the identification

$$
\mu \mapsto \mu(f), \quad \mu \in \mathcal{M}(K) .
$$

Thus $\mathcal{U}^{b}(K) \cap \mathcal{H}^{\perp \perp}$ is the space of all bounded universally measurable functions $f$ on $K$, for which $\mu(f)=0$ for every $\mu \in \mathcal{H}^{\perp}$. In the sequel, we will use this identification frequently.

If $\varphi: K \rightarrow L$ is a continuous mapping and $\mu \in \mathcal{M}(K)$, then $\varphi \mu \in \mathcal{M}(L)$ denotes the image of $\mu$. 
If $X$ is a Banach space, $B_{X}$ denotes its unit ball. A Banach space $X$ is complemented in some $\mathcal{C}(K)$-space if there exists a compact space $K$ and an isomorphism $T: X \rightarrow \mathcal{C}(K)$ such that $T X$ is complemented in $\mathcal{C}(K)$.

If $\mathcal{F}$ is a family of functions on a set $K$, then we write $\mathcal{F}^{b}$ for the set of all bounded functions in $\mathcal{F}$.

For a function $f$ on a set $K$ and $a \in \mathbb{R}$ we denote by $[f>a]$ the level set $\{x \in K: f(x)>a\}$. The meaning of $[f=a],[f<a]$, etc., should be clear. The characteristic function of a set $A$ is denoted by $\chi_{A}$, the restriction of a function $f$ to a set $A$ is denoted by $\left.f\right|_{A}$. If $f$ is a function, $\|f\|$ will always mean the sup-norm of $f$.

We recall the following fundamental property of Baire-one functions (see [2, Theorem I.3.1]).

Theorem 2.1. If $f: K \rightarrow \mathbb{R}$ is a Baire-one function on a completely metrizable space $K$, then the set of all points of continuity of $f$ is a dense $G_{\delta}$-subset of $K$.

2.1. Function spaces. Throughout the paper we will consider a function space $\mathcal{H}$ on a compact space $K$. By this we mean a (not necessarily closed) linear subspace of $\mathcal{C}(K)$ containing the constant functions and separating the points of $K$. Let $\mathcal{M}_{x}(\mathcal{H})$ be the set of all $\mathcal{H}$-representing measures for $x \in K$, i.e.,

$$
\mathcal{M}_{x}(\mathcal{H})=\left\{\mu \in \mathcal{M}^{1}(K): f(x)=\int_{K} f d \mu \text { for any } f \in \mathcal{H}\right\} .
$$

If $\mu \in \mathcal{M}_{x}(\mathcal{H})$, we say that $x$ is an $\mathcal{H}$-barycenter of $\mu$ and denote $x=r(\mu)$.

The set

$$
\mathrm{Ch}_{\mathcal{H}} K=\left\{x \in K: \mathcal{M}_{x}(\mathcal{H})=\left\{\varepsilon_{x}\right\}\right\}
$$

is called the Choquet boundary of $\mathcal{H}$. It may be highly irregular from the topological point of view but it is a $G_{\delta}$-set if $K$ is metrizable (see [1, Corollary I.5.17]).

We say that a function $h \in \mathcal{H}$ is $\mathcal{H}$-exposing for $x \in K$ if $h$ attains its minimum precisely at $x$. Obviously, any $\mathcal{H}$-exposed point is contained in the Choquet boundary of $\mathcal{H}$.

2.2. Examples of function spaces. We introduce the following main examples of function spaces.

In the "convex case", the function space $\mathcal{H}$ is the linear space $\mathfrak{A}(X)$ of all continuous affine functions on a compact convex subset $X$ of a locally convex space. In this example, the Choquet boundary of $\mathfrak{A}(X)$ coincides with the set of all extreme points of $X$ (see [3, Theorem 6.3]) and is denoted by ext $X$.

Further, the barycenter of a probability measure $\mu$ on $X$ is the unique point $r(\mu) \in X$ for which $f(r(\mu))=\mu(f)$ for any $f \in \mathfrak{A}(X)$; in other words, $x$ is $\mathfrak{A}(X)$ represented by $\mu$.

In the "harmonic case", $U$ is a bounded open subset of the Euclidean space $\mathbb{R}^{m}$ and the corresponding function space $\mathcal{H}$ is $\mathbf{H}(U)$, i.e., the family of all continuous functions on $\bar{U}$ which are harmonic on $U$. In the "harmonic case", the Choquet boundary of $\mathbf{H}(U)$ coincides with the set $\partial_{\text {reg }} U$ of all regular points of $U$ (see [21, Theorem, p. 625]).

2.3. $\mathcal{H}$-affine functions. We define the space $\mathcal{A}(\mathcal{H})$ of all $\mathcal{H}$-affine functions as the family of all bounded universally measurable functions $f$ on $K$ satisfying

$$
f(x)=\int_{K} f d \mu \quad \text { for each } \quad x \in K \quad \text { and } \quad \mu \in \mathcal{M}_{x}(\mathcal{H}) .
$$


Further, let $\mathcal{A}_{c}(\mathcal{H})$ be the family of all continuous $\mathcal{H}$-affine functions on $K$. Then $\mathcal{A}_{c}(\mathcal{H})$ is a uniformly closed function space with $\mathcal{M}_{x}(\mathcal{H})=\mathcal{M}_{x}\left(\mathcal{A}_{c}(\mathcal{H})\right)$ for every $x \in K$. It is easy to deduce that $\mathcal{A}_{c}(\mathcal{H})$ coincides with $\mathcal{H}$ in both the "convex" and "harmonic" cases.

For any countable ordinal $\alpha$ we define functions of $\mathcal{H}$-affine class $\alpha$ as follows: we set $\mathcal{H}_{0}=\mathcal{H}$ and having $\mathcal{H}_{\beta}, \beta<\alpha$, already defined for an ordinal number $\alpha \in\left(0, \omega_{1}\right)$, we define $\mathcal{H}_{\alpha}$ to be the space of all pointwise limits of bounded sequences of functions from $\bigcup_{\beta<\alpha} \mathcal{H}_{\beta}$.

If we take $\mathcal{H}$ to be the space $\mathcal{C}(K)$ of all continuous functions on $K,(\mathcal{C}(K))_{\alpha}$ is the usual space $\mathcal{B}_{\alpha}^{b}(K)$ of all bounded Baire $-\alpha$ functions on $K$.

We remark that this definition coincides in the "convex case" with the definition of functions of affine class $\alpha$ mentioned in the introduction. The aforementioned results of G. Choquet and G. Mokobodzki can be used to show that $\mathcal{B}_{1}^{b}(K) \cap \mathcal{H}^{\perp \perp}=$ $\mathcal{H}_{1}$ (see Theorem 2.5 below or [17, Theorem 5.1]).

2.4. Simplicial function spaces. The convex cone $\mathcal{K}_{c}(\mathcal{H})$ of all continuous $\mathcal{H}-$ convex functions (i.e., continuous functions $f$ satisfying $\mu(f) \geq f(x)$ for each $\mu \in$ $\mathcal{M}_{x}(\mathcal{H})$ and $x \in K$ ) determines a partial ordering $\preceq$ (called the Choquet ordering) on the space $\mathcal{M}^{+}(K)$ of all positive Radon measures on $K$ :

$$
\mu \preceq \nu \quad \text { if } \quad \mu(f) \leq \nu(f) \quad \text { for each } \quad f \in \mathcal{K}_{c}(\mathcal{H}) .
$$

For any point $x \in K$, the Choquet-Bishop-deLeeuw theorem provides an $\mathcal{H}$ maximal measure $\mu \in \mathcal{M}^{1}(K)$ (i.e., maximal with respect to $\preceq$ ) that $\mathcal{H}$-represents $x$ (see e.g. 11, Theorem I.4.8]).

If $K$ is metrizable, then a measure $\mu \in \mathcal{M}^{1}(K)$ is $\mathcal{H}$-maximal if and only if $\mu\left(K \backslash \mathrm{Ch}_{\mathcal{H}} K\right)=0$ (see [1, Corollary I.5.18]).

If an $\mathcal{H}$-maximal measure $\mathcal{H}$-representing $x \in K$ is uniquely determined for every $x \in K$, we say that $\mathcal{H}$ is a simplicial function space. In the "convex case" it is equivalent to saying that $X$ is a Choquet simplex, briefly simplex (see [1, Theorem II.3.6], [3, Theorem 7.3] or [11, Section 3]).

For another example of a simplicial function space, consider the space $\mathbf{H}(U)$ from the "harmonic case" (see e.g. 21, Theorem, p. 625]).

For a simplicial function space $\mathcal{H}$ and $x \in K$ we denote the unique $\mathcal{H}$-maximal measure $\mathcal{H}$-representing $x$ by $\delta_{x}$.

2.5. Embeddings of $\mathcal{H}$-affine functions. If $\mathcal{H}$ is a function space on a compact space $K$, let $X$ stand for the dual unit ball $B_{\mathcal{H}^{*}}$ endowed with the weak ${ }^{*}$-topology and let $\pi: B_{\mathcal{M}(K)} \rightarrow X$ be the restriction mapping. Let $\phi: K \rightarrow X$ be the evaluation mapping, i.e., $\phi(x)(h)=h(x), h \in \mathcal{H}, x \in K$. In other words, $\phi(x)=$ $\pi\left(\varepsilon_{x}\right)$. Then $\phi$ is a homeomorphism of $K$ into $B_{\mathcal{H}^{*}}$.

We define

$$
\begin{gathered}
I: \mathcal{U}^{b}(K) \cap \mathcal{H}^{\perp \perp} \rightarrow \mathfrak{A}_{\text {odd }}(X), \\
I f(s)=\mu(f), \quad \mu \in \pi^{-1}(s) .
\end{gathered}
$$

Then $I$ is an extension of the canonical embedding of $\mathcal{H}$ into $\mathcal{H}^{* *}$. In order to get properties of $I$ needed for our purposes, we have to establish several lemmas.

Lemma 2.2. Let $\varphi: X_{1} \rightarrow X_{2}$ be a continuous mapping of a compact space $X_{1}$ onto a compact space $X_{2}$ and let $f$ be a function on $X_{2}$. 
Then the function $f \circ \varphi$ is universally measurable (Borel, of Baire class $\alpha$, respectively) on $X_{1}$ if and only if $f$ is universally measurable (Borel, of Baire class $\alpha$, respectively) on $X_{2}$.

Proof. See [24, Theorem 2.1].

Lemma 2.3. Let $\varphi: X_{1} \rightarrow X_{2}$ be a continuous affine mapping of a compact convex set $X_{1}$ onto a compact convex set $X_{2}$ and let $f$ be a bounded function on $X_{2}$.

Then $f \circ \varphi \in \mathfrak{A}_{\mathrm{bf}}\left(\mathrm{X}_{1}\right)$ if and only if $f \in \mathfrak{A}_{\mathrm{bf}}\left(\mathrm{X}_{2}\right)$.

Proof. See [24, Proposition 3.2].

Lemma 2.4. Let $F_{1}, F_{2}$ be closed convex subsets of a compact convex set $X$ such that $\operatorname{co}\left(F_{1} \cup F_{2}\right)=X$. Let $f$ be an affine function on $X$.

(a) The function $f$ is universally measurable (Borel, of Baire class $\alpha$, respectively) on $X$ if and only if $f$ is universally measurable (Borel, of Baire class $\alpha$, respectively) on $F_{1} \cup F_{2}$;

(b) $f \in \mathfrak{A}_{\mathrm{bf}}(\mathrm{X})$ if and only if $\left.f\right|_{F_{1}} \in \mathfrak{A}_{\mathrm{bf}}\left(\mathrm{F}_{1}\right)$ and $\left.f\right|_{F_{2}} \in \mathfrak{A}_{\mathrm{bf}}\left(\mathrm{F}_{2}\right)$.

Proof. We set $Y=F_{1} \times F_{2} \times[0,1]$ and define a continuous surjection

$$
\begin{aligned}
\varphi: & Y \rightarrow X, \\
& \left(x_{1}, x_{2}, \lambda\right) \mapsto \lambda x_{1}+(1-\lambda) x_{2} .
\end{aligned}
$$

Let $\widehat{f}: Y \rightarrow \mathbb{R}$ be defined as

$$
\widehat{f}\left(x_{1}, x_{2}, \lambda\right)=\lambda f\left(x_{1}\right)+(1-\lambda) f\left(x_{2}\right), \quad\left(x_{1}, x_{2}, \lambda\right) \in Y .
$$

Then $\widehat{f}$ inherits topological properties of $\left.f\right|_{F_{1} \cup F_{2}}$ and $\widehat{f}=f \circ \varphi$. Thus Lemma 2.2 yields (a).

For the proof of the nontrivial part of (b), let $f$ be an affine function on $X$ such that $\left.f\right|_{F_{i}} \in \mathfrak{A}_{\mathrm{bf}}\left(\mathrm{F}_{i}\right), i=1,2$. Then $f$ is bounded on $F_{1} \cup F_{2}$, and thus also on $F$. By (a), $f$ is universally measurable on $X$.

Let $x \in X$ and $\mu \in \mathcal{M}^{1}(\overline{\operatorname{ext} X})$ with $r(\mu)=x$ being given. As $X \subset \operatorname{co}\left(F_{1} \cup F_{2}\right)$, Milman's theorem yields $\overline{\operatorname{ext} X} \subset F_{1} \cup F_{2}$ (see [23, Theorem 3.25]). We set

$$
a_{1}=\mu\left(F_{1}\right) \quad \text { and } \quad a_{2}=\mu\left(F_{2} \backslash F_{1}\right) .
$$

Obviously, we may assume that $a_{1}, a_{2}>0$. We define

$$
\mu_{1}=\left.a_{1}^{-1} \mu\right|_{F_{1}} \quad \text { and } \quad \mu_{2}=\left.a_{2}^{-1} \mu\right|_{F_{2} \backslash F_{1}} .
$$

Then $r\left(\mu_{i}\right) \in F_{i}, i=1,2$,

$$
a_{1}+a_{2}=1 \quad \text { and } \quad a_{1} r\left(\mu_{1}\right)+a_{2} r\left(\mu_{2}\right)=x .
$$

According to the assumption,

$$
\mu(f)=a_{1} \mu_{1}(f)+a_{2} \mu_{2}(f)=a_{1} f\left(r\left(\mu_{1}\right)\right)+a_{2} f\left(r\left(\mu_{2}\right)\right)=f(x) .
$$

Thus $f$ satisfies the barycentric formula with respect to any measure supported by $\overline{\operatorname{ext} X}$. According to [24, Theorem 3.3], $f \in \mathfrak{A}_{\mathrm{bf}}(\mathrm{X})$. This concludes the proof.

Theorem 2.5. Let $\mathcal{H}$ be a closed function space on a compact space $K$ and $X=$ $B_{\mathcal{H}^{*}}$. Then the mapping I has the following properties:

(a) $I$ is an isometric isomorphism of $\mathcal{U}^{b}(K) \cap \mathcal{H}^{\perp \perp}$ onto $\mathfrak{A}_{\mathrm{bf}}(\mathrm{X}) \cap \mathfrak{A}_{\text {odd }}(\mathrm{X})$;

(b) $I^{-1}(F)=F \circ \phi, F \in \mathfrak{A}_{\mathrm{bf}}(\mathrm{X}) \cap \mathfrak{A}_{\text {odd }}(\mathrm{X})$;

(c) $I\left(\mathcal{B}^{b}(K) \cap \mathcal{H}^{\perp \perp}\right)=\mathcal{B}(X) \cap \mathfrak{A}_{\mathrm{bf}}(\mathrm{X}) \cap \mathfrak{A}_{\text {odd }}(\mathrm{X})$; 
(d) $I\left(\mathcal{B}_{\alpha}^{b}(K) \cap \mathcal{H}^{\perp \perp}\right)=\mathcal{B}_{\alpha}(X) \cap \mathfrak{A}_{\mathrm{bf}}(\mathrm{X}) \cap \mathfrak{A}_{\text {odd }}(\mathrm{X}), \alpha \in\left[0, \omega_{1}\right)$;

(e) if $\left\{f_{n}\right\} \subset \mathcal{U}(K)$ is a bounded sequence pointwise converging to $f$, then $I f_{n} \rightarrow$ If pointwise;

(f) $I\left(\mathcal{H}_{\alpha}\right)=\mathcal{H}_{\alpha}^{* *}, \alpha \in\left[0, \omega_{1}\right)$.

Proof. Obviously, $I$ is linear and $I\left(\mathcal{U}^{b}(K) \cap \mathcal{H}^{\perp \perp}\right) \subset \mathfrak{A}_{\text {odd }}(X)$. The equalities

$$
\|I f\|=\sup _{s \in X}|I f(s)|=\sup _{\mu \in B_{\mathcal{M}(K)}}|\mu(f)|=\|f\|
$$

show that $I$ is an isometry.

Let $\widehat{I}: \mathcal{U}^{b}(K) \rightarrow \mathcal{U}^{b}\left(B_{\mathcal{M}(K)}\right)$ be defined as

$$
\widehat{I} f(\mu)=\mu(f), \quad \mu \in B_{\mathcal{M}(K)} .
$$

First, we check that $\widehat{I}$ maps $\mathcal{U}^{b}(K)$ into $\mathcal{U}^{b}\left(B_{\mathcal{M}(K)}\right)$.

If $f \in \mathcal{U}^{b}(K)$, then [24, Proposition 3.1] yields that $\left.\widehat{I} f\right|_{\mathcal{M}^{1}(K)}$ is universally measurable and satisfies the barycentric formula. Moreover, $\widehat{I} f$ is Borel or of Baire class $\alpha$, respectively, if $f$ is Borel or of Baire class $\alpha$, respectively.

By symmetry, $\left.\widehat{I} f\right|_{-\mathcal{M}^{1}(K)}$ satisfies analogous properties. Since

$$
B_{\mathcal{M}(K)}=\operatorname{co}\left(\mathcal{M}^{1}(K) \cup-\mathcal{M}^{1}(K)\right)
$$

Lemma 2.4 yields that $\widehat{I} f \in \mathfrak{A}_{\mathrm{bf}}\left(\mathrm{B}_{\mathcal{M}(K)}\right)$ and $\widehat{I} f$ is Borel (of Baire class $\alpha$, respectively) on $B_{\mathcal{M}(K)}$ if $f$ is Borel (of Baire class $\alpha$, respectively) on $K$.

Since

$$
\widehat{I} f(\mu)=I f(\pi(\mu)), \quad \mu \in B_{\mathcal{M}(K)}, f \in \mathcal{U}^{b}(K) \cap \mathcal{H}^{\perp \perp},
$$

Lemmata 2.2 and 2.3 imply the inclusions " $\subset$ " in (a), (c) and (d).

For the proof of (b), let $F \in \mathfrak{A}_{\mathrm{bf}}(\mathrm{X}) \cap \mathfrak{A}_{\text {odd }}(\mathrm{X})$ be given and let $f=F \circ \phi$. Then $f$ inherits all topological properties from $F$ because $\phi$ is a homeomorphism.

We pick a measure $\mu \in \mathcal{H}^{\perp}$. Without loss of generality, we may assume that $\mu=\mu_{1}-\mu_{2}, \mu_{1}, \mu_{2} \in \mathcal{M}^{1}(K)$. Then $r\left(\phi \mu_{i}\right)=\pi\left(\mu_{i}\right), i=1,2$ (see [24, Lemma 4.2]). Then

$$
\mu_{i}(f)=\mu_{i}(F \circ \phi)=\phi \mu_{i}(F)=F\left(r\left(\phi \mu_{i}\right)\right)=F\left(\pi\left(\mu_{i}\right)\right), \quad i=1,2 .
$$

Since $\pi\left(\mu_{1}\right)=\pi\left(\mu_{2}\right), \mu(f)=0$ and $f \in \mathcal{H}^{\perp \perp}$.

Further, given $s \in X$, we select a measure $\mu \in \pi^{-1}(s)$. Let $\mu=a \mu_{1}-(1-a) \mu_{2}$, $a \in[0,1], \mu_{1}, \mu_{2} \in \mathcal{M}^{1}(K)$. Using the affinity of $F$ and (3), we get

$$
\begin{aligned}
I f(s) & =\mu(f)=a \mu_{1}(f)-(1-a) \mu_{2}(f) \\
& =a F\left(\pi\left(\mu_{1}\right)\right)-(1-a) F\left(\pi\left(\mu_{2}\right)\right)=F(\pi(\mu)) \\
& =F(s) .
\end{aligned}
$$

Thus $I^{-1}(F)=F \circ \phi$ and we get the inclusions " $\supset$ " in (a), (c) and (d).

Using the Lebesgue dominated convergence theorem we get (e).

By transfinite induction and (e), $I\left(\mathcal{H}_{\alpha}\right)=\mathcal{H}_{\alpha}^{* *}$, which finishes the proof.

2.6. State space. If $\mathcal{H}$ is a closed function space on a compact space $K$ and we restrict the embedding $I$ from 2.5 to the set

$$
\mathbf{S}(\mathcal{H})=\left\{s \in \mathcal{H}^{*}:\|s\|=s(1)=1\right\},
$$

then we get the standard identification of $\mathcal{H}$ with $\mathfrak{A}(\mathbf{S}(\mathcal{H})$ ) (see e.g. 1, Chapter 2, $\S 2]$, [3, Chapter 1, §4] or [20, Section 6]). The set $\mathbf{S}(\mathcal{H})$ is called the state space of 
$\mathcal{H}$. The mapping $\phi$ defined above is a homeomorphic embedding of $K$ into $\mathbf{S}(\mathcal{H})$ and $\phi\left(\mathrm{Ch}_{\mathcal{H}} K\right)=\operatorname{ext} \mathbf{S}(\mathcal{H})$. Further, $\left.f \mapsto I f\right|_{\mathbf{S}(\mathcal{H})}$ is an isometric isomorphism of $\mathcal{U}^{b}(K) \cap \mathcal{H}^{\perp \perp}$ onto $\mathfrak{A}_{\mathrm{bf}}(\mathbf{S}(\mathcal{H}))$ that preserves the natural order of functions.

2.7. Properties of $\mathcal{H}$. In the sequel, we will need the following theorem collecting several results on simplicial function spaces.

Theorem 2.6. Let $\mathcal{H}$ be a function space on a compact space $K$.

(a) If $K$ is metrizable and $\mathcal{H}$ is closed, then the following assertions are equivalent:

(i) $\mathcal{H}$ is simplicial and $\mathcal{A}_{c}(\mathcal{H})=\mathcal{H}$;

(ii) for any compact set $F \subset \mathrm{Ch}_{\mathcal{H}} K$ and $f \in \mathcal{C}(F)$ there exists $h \in \mathcal{H}$ such that $f=h$ on $F$ and $\|f\|=\|h\|$;

(iii) $\mathcal{H}$ satisfies the weak Riesz interpolation property (that is, whenever functions $f_{1}, f_{2}, g_{1}, g_{2} \in \mathcal{H}$ satisfy $\max \left\{f_{1}, f_{2}\right\}<\min \left\{g_{1}, g_{2}\right\}$, then there exists a function $h \in \mathcal{H}$ with $\left.\max \left\{f_{1}, f_{2}\right\}<h<\min \left\{g_{1}, g_{2}\right\}\right)$;

(iv) the state space $\mathbf{S}(\mathcal{H})$ is a simplex.

(b) Let $\mathcal{H}$ be simplicial.

(b1) if $K$ is metrizable, then any point of $\mathrm{Ch}_{\mathcal{H}} K$ is $\mathcal{A}_{c}(\mathcal{H})$-exposed;

(b2) $\mathcal{A}(\mathcal{H})=\mathcal{U}^{b}(K) \cap\left(\mathcal{A}_{c}(\mathcal{H})\right)^{\perp \perp}$;

(b3) if a function $f \in \mathcal{U}^{b}(K)$ satisfies $\delta_{x}(f)=f(x)$ for each $x \in K$, then $f$ is $\mathcal{H}$-affine.

Proof. For the proof of (i) $\Longrightarrow$ (iv) in (a), see [6. Theorem]. By [1, Corollary II.3.11, Theorem II.3.12] and properties of $I$, (ii) $\Longleftrightarrow$ (iii) $\Longleftrightarrow$ (iv).

To close the chain of implications, we first notice that $\mathcal{H}$ is simplicial provided (iii) holds. This follows from (ii) (which is equivalent with (iii)) exactly as in the proof of [1, Theorem II.3.12]. Thus we must verify that $\mathcal{A}_{c}(\mathcal{H})=\mathcal{H}$.

To this end, let $f \in \mathcal{A}_{c}(\mathcal{H})$ be given. Then for $x \in K$,

$$
\begin{aligned}
f(x) \leq f^{*}(x) & =\inf \{h(x): h \in \mathcal{H}, h \geq f\} \\
& =\sup \left\{\mu(f): \mu \in \mathcal{M}_{x}(\mathcal{H})\right\} \\
& =f(x),
\end{aligned}
$$

where the first equality is just the definition of $f^{*}$ and the second one follows by [1, Proposition I.5.8].

Fix $\varepsilon>0$. An easy compactness argument provides functions $f_{1}, \ldots, f_{n} \in \mathcal{H}$ such that $f<\min \left\{f_{1}, \ldots, f_{n}\right\}<f+\varepsilon$. Analogously, there exist functions $g_{1}, \ldots, g_{m} \in \mathcal{H}$ such that $f-\varepsilon<\max \left\{g_{1} \ldots, g_{m}\right\}<f$. We apply inductively the weak Riesz interpolation property to get a function $h \in \mathcal{H}$ such that

$$
\max \left\{g_{1}, \ldots, g_{m}\right\}<h<\min \left\{f_{1}, \ldots, f_{n}\right\} .
$$

Then $\|f-h\|<\varepsilon$. Since $\mathcal{H}$ is closed, $f$ belongs to $\mathcal{H}$, which concludes the proof of part (a).

Since the part (b1) follows by [1, Corollary II.5.21], [6, Theorem] and properties of $I$, we can proceed to (b2).

We need to check that $\mathcal{A}(\mathcal{H}) \subset \mathcal{U}^{b}(K) \cap\left(\mathcal{A}_{c}(\mathcal{H})\right)^{\perp \perp}$. Given a function $f \in \mathcal{A}(\mathcal{H})$ and a measure $\mu \in\left(\mathcal{A}_{c}(\mathcal{H})\right)^{\perp}$, let $\nu$ be the measure defined as

$$
\nu(g)=\int_{K} \delta_{x}(g) d \mu(x), \quad g \in \mathcal{C}(K) .
$$


According to [1, Corollary II.3.15 and Proposition I.5.9], $\nu$ is well defined and both the positive and negative parts of $\nu$ are $\mathcal{H}$-maximal measures. Since $\nu \in\left(\mathcal{A}_{c}(\mathcal{H})\right)^{\perp}$ and $\mathcal{H}$ is simplicial, $\nu=0$ by [18, Theorem 14.4]. Thus,

$$
0=\nu(f)=\mu(f)
$$

and $f \in\left(\mathcal{A}_{c}(\mathcal{H})\right)^{\perp \perp}$.

For the proof of (b3), let $f$ be as in the premise and $x \in K, \mu \in \mathcal{M}_{x}(\mathcal{H})$ be given. The measure

$$
\nu: g \mapsto \int_{K} \delta_{x}(g) d \mu(x), \quad g \in \mathcal{C}(K),
$$

is $\mathcal{H}$-maximal (use again [1, Proposition I.5.9]) and $\mathcal{H}$-represents $x$. Thus $\nu=\delta_{x}$ and

$$
f(x)=\delta_{x}(f)=\nu(f)=\mu(f) .
$$

Hence, $f \in \mathcal{A}(\mathcal{H})$ and we are done.

2.8. Spaces of sequences. We write $\mathbb{N}<\mathbb{N}$ for the set of all finite sequences of natural numbers, $\mathbb{N}^{\mathbb{N}}$ for infinite sequences of natural numbers and $|s|$ for the length of $s \in \mathbb{N}<\mathbb{N}$. If $s \in \mathbb{N}<\mathbb{N}$ and $n \in \mathbb{N}, s^{\wedge} n=\left(s_{1}, \ldots, s_{|s|}, n\right)$. If $\sigma \in \mathbb{N}^{\mathbb{N}}$ and $n \in \mathbb{N}$, $\left.\sigma\right|_{n}=\left(\sigma_{1}, \ldots, \sigma_{n}\right)$. For a couple of sequences $s, t \in \mathbb{N}<\mathbb{N}$, we write $s \subset t$ if $|s| \leq|t|$ and $\left.t\right|_{|s|}=s$.

Let $\{0,1\}^{\mathbb{N}}$ stand for the Cantor set endowed with the usual topology and let $\{0,1\}^{<\mathbb{N}}$ denote the set of all finite sequences of digits 0 and 1 . We adopt the same notation as above for sequences in $\{0,1\}^{\mathbb{N}}$ or in $\{0,1\}^{<\mathbb{N}}$. Given a sequence $t \in\{0,1\}^{<\mathbb{N}}$, let $U_{t}$ denote the standard clopen base set in $\{0,1\}^{\mathbb{N}}$, i.e.,

$$
U_{t}=\left\{\tau \in\{0,1\}^{\mathbb{N}}:\left.\tau\right|_{|t|}=t\right\} .
$$

We also denote as $\mu_{t}$ the probability measure supported by $U_{t}$ that satisfies

$$
\mu_{t}\left(U_{s}\right)=\left\{\begin{array}{ll}
2^{-(|s|-|t|)}, & t \subset s, \\
0, & \text { otherwise, }
\end{array} \quad s \in\{0,1\}^{<\mathbb{N}},|s| \geq|t| .\right.
$$

In particular, $\mu_{\emptyset}$ is the usual product measure on $\{0,1\}^{\mathbb{N}}$. We remark that, for each $t \in\{0,1\}^{<\mathbb{N}}$ and $n \geq|t|$,

$$
\mu_{t}=\sum_{t \subset s,|s|=n} 2^{-(n-|t|)} \mu_{s} .
$$

\section{Auxiliary topological Results}

We recall that a Polish space means a separable completely metrizable space; its perfect subset is a compact set without isolated points. We start auxiliary topological lemmas with the following standard result that can be easily deduced from [14, Theorem 6.2].

Lemma 3.1. Let $K$ be a nonempty Polish space without isolated points. Then there exists a nonempty nowhere dense perfect set $C \subset K$.

Lemma 3.2. Let $(K, \rho)$ be a nonempty separable complete metric space without isolated points and let $\left\{r_{n}\right\}$ be a sequence of positive numbers. Then there exists a countable family $\left\{C_{n}: n \in \mathbb{N}\right\}$ of pairwise disjoint nonempty perfect nowhere dense subsets of $K$ such that $\bigcup_{n} C_{n}$ is dense in $K$ and $\operatorname{diam} C_{n}<r_{n}$. 
Proof. Let $\left\{U_{n}: n \in \mathbb{N}\right\}$ be a countable base of open sets of $K$. Let $V_{n}, n \in \mathbb{N}$, be nonempty open sets such that $V_{n} \subset U_{n}$ and diam $V_{n}<r_{n}$. Using Lemma 3.1 we inductively find nonempty perfect nowhere dense sets $C_{n} \subset V_{n}$ such that $C_{n+1}$ is disjoint with $C_{1} \cup \cdots \cup C_{n}$. Then the family $\left\{C_{n}: n \in \mathbb{N}\right\}$ possesses the required properties.

An inductive use of Lemma 3.2 provides the following well-known construction (see [5, [19, pp. 82-86] or [12, Lemma 2.3]).

Lemma 3.3. Let $K$ be a nonempty separable complete metric space without isolated points. Then there exists a family $\left\{F_{s}: s \in \mathbb{N}^{<\mathbb{N}}\right\}$ such that

(a) $F_{\emptyset}=K$;

(b) $\left\{F_{s^{\wedge} n}: n \in \mathbb{N}\right\}$ is a disjoint family of nonempty nowhere dense perfect subsets of $F_{s}$;

(c) $\bigcup\left\{F_{s^{\wedge} n}: n \in \mathbb{N}\right\}$ is dense in $F_{s}$;

(d) $\operatorname{diam} F_{s}<2^{-\left(s_{1}+\cdots+s_{|s|}\right)}, s \in \mathbb{N}<\mathbb{N}$.

If $K$ is a metrizable space and $\alpha \in\left[1, \omega_{1}\right)$, we recall that $\boldsymbol{\Sigma}_{\alpha}^{0}(K)\left(\boldsymbol{\Pi}_{\alpha}^{0}(K)\right.$, $\boldsymbol{\Delta}_{\alpha}^{0}(K)$, respectively) is the family of all Borel sets in $K$ of additive (multiplicative, ambiguous, respectively) class $\alpha$ (see 14, Chapter II, Section 11.a]).

We sometimes write $F_{\sigma}\left(\right.$ respectively $\left.G_{\delta}\right)$ instead of $\boldsymbol{\Sigma}_{2}^{0}(K)$ (respectively $\boldsymbol{\Pi}_{2}^{0}(K)$ ).

Lemma 3.4. Let $\left\{A_{n}\right\} \subset \boldsymbol{\Sigma}_{3}^{0}(K)$ be a sequence of sets in a metrizable space $K$ such that $\bigcup_{n} A_{n} \in \boldsymbol{\Delta}_{3}^{0}(K)$. Let $f$ be a function on $K$ such that $f \in \mathcal{B}_{2}\left(A_{n}\right), n \in \mathbb{N}$, and $f \in \mathcal{B}_{2}\left(K \backslash \bigcup_{n} A_{n}\right)$. Then $f \in \mathcal{B}_{2}(K)$.

Proof. Let $f$ be as in the premise and let $U \subset \mathbb{R}$ be open. By the assumption,

$$
f^{-1}(U)=\bigcup_{n=1}^{\infty}\left(f^{-1}(U) \cap A_{n}\right) \cup\left(f^{-1}(U) \cap\left(K \backslash \bigcup_{n=1}^{\infty} A_{n}\right)\right)
$$

is in $\boldsymbol{\Sigma}_{3}^{0}(K)$. According to the Lebesgue-Hausdorff characterization of Baire functions (see [14, Theorem 24.3]), $f \in \mathcal{B}_{2}(K)$.

Lemma 3.5. For $i=1, \ldots, n$, let $G_{i}$ be a dense $G_{\delta}$-subset of a completely metrizable space $K, a_{i} \in \mathbb{R}$ and let $\left\{f_{i k}\right\}$ be a sequence of Baire-one functions on $K$ such that $f_{i k} \rightarrow a_{i} \chi_{G_{i}}$.

Then, for every $\varepsilon>0$ there exists $k_{0} \in \mathbb{N}$ such that for each $k \geq k_{0}$ there exists a nonempty open set $V \subset K$ such that $f_{i k}(V) \subset\left(a_{i}-\varepsilon, a_{i}+\varepsilon\right), i=1, \ldots, n$.

Proof. Given objects as in the premise, let $A_{i k}$ be the set of all points of continuity of $f_{i k}, k \in \mathbb{N}, i=1, \ldots, n$. By Theorem 2.1 and our assumption on the sets $G_{i}$, $i=1, \ldots, n$, we can select a point

$$
x \in \bigcap_{i=1}^{n} G_{i} \cap \bigcap\left\{A_{i k}: i=1, \ldots, n, k \in \mathbb{N}\right\} .
$$

Given $\varepsilon>0$, let $k_{0} \in \mathbb{N}$ be chosen such that $f_{i k}(x) \in\left(a_{i}-\varepsilon, a_{i}+\varepsilon\right)$ for each $k \geq k_{0}$ and $i=1, \ldots, n$. As $x$ is a point of continuity of all functions $f_{i k}$, to finish the proof it is enough to find for a given $k \geq k_{0}$ a suitable neighborhood $V \subset U$ of $x$ such that $f_{i k}(V) \subset\left(a_{i}-\varepsilon, a_{i}+\varepsilon\right), i=1, \ldots, n$. 
Lemma 3.6. Assume that $\left\{f_{n}\right\}$ is a bounded sequence of $\mu_{\emptyset}$-measurable functions on $\{0,1\}^{\mathbb{N}}$. Let $0<\varepsilon^{\prime}<\varepsilon$ and $\left\{a_{n}^{t}: n \in \mathbb{N}, t \in\{0,1\}^{n}\right\}$ be real numbers such that

- $\left|\mu_{t}\left(f_{n}\right)-a_{n}^{t}\right|<\varepsilon^{\prime}$, and

- $\varepsilon<\left|a_{n}^{t}\right|, n \in \mathbb{N}, t \in\{0,1\}^{n}$.

Then $f_{n} \not \rightarrow 0 \mu_{\emptyset}$-almost everywhere on $\{0,1\}^{\mathbb{N}}$.

Proof. Assume that there exists a sequence $\left\{f_{n}\right\}$ of functions on $\{0,1\}^{\mathbb{N}}$ satisfying the hypothesis such that $f_{n} \rightarrow 0 \mu_{\emptyset}$-almost everywhere on $\{0,1\}^{\mathbb{N}}$.

We find $c>0$ such that $\sup _{n}\left\|f_{n}\right\| \leq c$ and $\delta \in\left(0, \varepsilon-\varepsilon^{\prime}\right)$.

Let $n \in \mathbb{N}$ be fixed. We set

$$
A_{n}=\left\{x \in\{0,1\}^{\mathbb{N}}:\left|f_{n}(x)\right| \geq \delta\right\} .
$$

Then, for each $t \in\{0,1\}^{n}$,

$$
\begin{aligned}
\varepsilon<\left|a_{n}^{t}\right| & \leq\left|a_{n}^{t}-\mu_{t}\left(f_{n}\right)\right|+\left|\mu_{t}\left(f_{n}\right)\right| \\
& \leq \varepsilon^{\prime}+\int_{A_{n}}\left|f_{n}\right| d \mu_{t}+\int_{\{0,1\}^{\mathbb{N}} \backslash A_{n}}\left|f_{n}\right| d \mu_{t} \\
& \leq \varepsilon^{\prime}+c \mu_{t}\left(A_{n}\right)+\delta .
\end{aligned}
$$

Thus

$$
\mu_{t}\left(A_{n}\right) \geq \frac{1}{c}\left(\varepsilon-\varepsilon^{\prime}-\delta\right) .
$$

Hence, for every $n \in \mathbb{N}$,

$$
\mu_{\emptyset}\left(A_{n}\right)=\sum_{|t|=n} \frac{1}{2^{n}} \mu_{t}\left(A_{n}\right) \geq \frac{1}{c}\left(\varepsilon-\varepsilon^{\prime}-\delta\right) .
$$

By the Lebesgue dominated convergence theorem, $\mu_{\emptyset}\left(\left|f_{n}\right|\right) \rightarrow 0$. But this is impossible by virtue of (4). This contradiction concludes the proof.

\section{Auxiliary Results on Baire classes of Banach spaces}

Proposition 4.1. Assume that $\mathcal{H} \subset \mathcal{C}(K)$ is a complemented subspace. Then $\mathcal{H}_{\alpha}=\mathcal{B}_{\alpha}^{b}(K) \cap \mathcal{H}^{\perp \perp}$ for every countable ordinal $\alpha$.

Proof. Let $P: \mathcal{C}(K) \rightarrow \mathcal{H}$ be a projection. For each $x \in K$ there exists a signed measure $\mu_{x} \in \mathcal{M}(K)$ such that $\left\|\mu_{x}\right\| \leq\|P\|$ and

$$
\mu_{x}(f)=P f(x), \quad f \in \mathcal{C}(K) .
$$

Then we can use (5) to define a mapping $P$ (denoted likewise) as $P f(x)=\mu_{x}(f)$, $f \in \mathcal{B}_{\omega_{1}}^{b}(X)$. It is straightforward to verify that $P$ is a projection of $\mathcal{B}_{\alpha}^{b}(K)$ onto $\mathcal{H}_{\alpha}$.

If $f \in \mathcal{B}_{\alpha}^{b}(K) \cap \mathcal{H}^{\perp \perp}$, then $P f \in \mathcal{H}_{\alpha}$. As $\mu_{x}-\varepsilon_{x} \in \mathcal{H}^{\perp}$ for each $x \in K, P f=f$ and we are done.

Proposition 4.2. Let $X$ be a Banach space that is complemented in some $\mathcal{C}(L)$ space. Then $X_{\alpha}^{* *}=X_{\mathcal{B}_{\alpha}}^{* *}$ for every countable ordinal $\alpha$.

Proof. Given a Banach space $X$, let $K=B_{X^{*}}$ considered with the weak*-topology and $\mathcal{H}=X \subset \mathcal{C}(K)$. Since $X$ is complemented in some $\mathcal{C}(L)$-space, [7, Lemma] yields that $\mathcal{H}$ is complemented in $\mathcal{C}(K)$. As

$$
X_{\mathcal{B}_{\alpha}}^{* *}=\mathcal{B}_{\alpha}^{b}(K) \cap \mathcal{H}^{\perp \perp},
$$


from Proposition 4.1 we get

$$
X_{\mathcal{B}_{\alpha}}^{* *}=\mathcal{H}_{\alpha}=X_{\alpha}^{* *}
$$

This finishes the proof.

Proposition 4.3. Let $\mathcal{H}$ be a function space on a compact space $K$ that is complemented in some $\mathcal{C}(L)$-space. Then $\mathcal{H}_{\alpha}=\mathcal{B}_{\alpha}^{b}(K) \cap \mathcal{H}^{\perp \perp}$ and $\mathcal{H}_{\alpha}^{* *}=\mathcal{H}_{\mathcal{B}_{\alpha}}^{* *}$ for every countable ordinal $\alpha$.

Proof. If $\mathcal{H}$ is complemented in a $\mathcal{C}(L)$-space, then $\mathcal{H}$ is closed and $\mathcal{H}_{\alpha}^{* *}=\mathcal{H}_{\mathcal{B}_{\alpha}}^{* *}$ according to Proposition 4.2. By Theorem 2.5.

$$
I\left(\mathcal{H}_{\alpha}\right)=\mathcal{H}_{\alpha}^{* *}=\mathcal{H}_{\mathcal{B}_{\alpha}}^{* *}=I\left(\mathcal{B}_{\alpha}^{b}(K) \cap \mathcal{H}^{\perp \perp}\right) .
$$

Thus, $\mathcal{H}_{\alpha}=\mathcal{B}_{\alpha}^{b}(K) \cap \mathcal{H}^{\perp \perp}$.

\section{Construction of a simplicial function space}

5.1. Key step. Let $\mathcal{H}_{1}$ be a simplicial function space on a metrizable compact space $K_{1}$ such that $\mathcal{A}_{c}\left(\mathcal{H}_{1}\right)=\mathcal{H}_{1}$. Let $\left\{F_{k}: k \in \mathbb{N}\right\}$ be a pairwise disjoint family of compact subsets of $\mathrm{Ch}_{\mathcal{H}_{1}} K_{1}$. We define a set $K_{2} \subset K_{1} \times \mathbb{R}$ as

$$
K_{2}=\left(K_{1} \times\{0\}\right) \cup \bigcup_{k=1}^{\infty}\left(F_{k} \times\{1 / k\}\right) \cup\left(F_{k} \times\{-1 / k\}\right) .
$$

If $p: K_{2} \rightarrow K_{1}$ denotes the natural projection, then $p$ is continuous and surjective and $K_{1}$ can be considered as a subspace of $K_{2}$ via the mapping $x \mapsto(x, 0), x \in K_{1}$. We define

$$
\begin{aligned}
& \mathcal{H}_{2}=\left\{f \in \mathcal{C}\left(K_{2}\right):\left.f\right|_{K_{1}} \in \mathcal{H}_{1}\right. \text { and } \\
&\left.f(x, 0)=\frac{1}{2}(f(x, 1 / k)+f(x,-1 / k)), x \in F_{k}, k \in \mathbb{N}\right\} .
\end{aligned}
$$

Lemma 5.1. Let $\left(K_{2}, \mathcal{H}_{2}\right)$ be constructed as above. Then

(a) $K_{2}$ is metrizable and $\mathcal{H}_{2}$ is a well-defined function space on $K_{2}$;

(b) the mapping $h \mapsto h \circ p, h \in \mathcal{H}_{1}$, provides an isometric embedding of $\mathcal{H}_{1}$ into $\mathcal{H}_{2}$

(c) $\mathcal{H}_{2}=\mathcal{A}_{c}\left(\mathcal{H}_{2}\right)$;

(d) the following holds:

$$
\mathrm{Ch}_{\mathcal{H}_{2}} K_{2}=\left(K_{2} \backslash K_{1}\right) \cup\left(\mathrm{Ch}_{\mathcal{H}_{1}} K_{1} \backslash \bigcup_{k=1}^{\infty} F_{k}\right) ;
$$

(e) $\mathcal{H}_{2}$ is simplicial;

(f) if $f$ is a bounded universally measurable function on $K_{2}$ such that $\left.f\right|_{K_{1}} \in$ $\mathcal{A}\left(\mathcal{H}_{1}\right)$ and $f$ satisfies the equations in (6), then $f \in \mathcal{A}\left(\mathcal{H}_{2}\right)$.

Proof. First, we prove (a) and (b). We notice that $K_{2}$ is indeed a compact metrizable space, $p: K_{2} \rightarrow K_{1}$ is a continuous surjective mapping, $K_{1}$ is homeomorphically embedded in $K_{2}$, and $\mathcal{H}_{1}$ is isometrically embedded in $\mathcal{H}_{2}$ via the mapping $p$.

Further, $\mathcal{H}_{2}$ contains constant functions and separates points of $K_{1}$ as it contains $\mathcal{H}_{1}$. We also get that $\mathcal{H}_{2}$ separates any couple of points in $K_{2}$ with distinct first coordinates. 
Let $x \in K_{1}$ be a point contained in some $F_{k}$. Then the function

$$
h= \begin{cases}1, & \text { on } F_{k} \times\{1 / k\} \\ -1, & \text { on } F_{k} \times\{-1 / k\} \\ 0, & \text { otherwise }\end{cases}
$$

is a function from $\mathcal{H}_{2}$ that separates points $(x, 0),(x, 1 / k)$ and $(x,-1 / k)$. Hence $\mathcal{H}_{2}$ separates points of $K_{2}$.

For the proof of (c), let $h \in \mathcal{A}_{c}\left(\mathcal{H}_{2}\right)$ be given. Then $\left.h\right|_{K_{1}} \in \mathcal{A}_{c}\left(\mathcal{H}_{1}\right)=\mathcal{H}_{1}$. Also $h$ obviously satisfies equalities (6) defining the space $\mathcal{H}_{2}$. Hence $h \in \mathcal{H}_{2}$.

Next, we verify $(\mathrm{d})$. We notice that for any point $x$ not contained in the righthand side of (7) there exists an $\mathcal{H}_{2}$-representing measure distinct from the Dirac measure $\varepsilon_{x}$. Thus we get the inclusion " $\subseteq$ ".

For the proof of the converse inclusion, let $x \in \mathrm{Ch}_{\mathcal{H}_{1}} K_{1} \backslash \bigcup_{k} F_{k}$ be given. By Theorem 2.6(b), there exists a function $h \in \mathcal{A}_{c}\left(\mathcal{H}_{1}\right)=\mathcal{H}_{1}$ that exposes $x$, i.e., $h \geq 0$ and $[h=0]=\{x\}$. Then, the function $h \circ p$ is in $\mathcal{H}_{2}$ and $\mathcal{H}_{2}$-exposes $x$. Thus, $x \in \mathrm{Ch}_{\mathcal{H}_{2}} K_{2}$.

If $x \in F_{k}$ for some $k \in \mathbb{N}$, let $g: F_{k} \rightarrow[0,1]$ be a continuous function satisfying $[g=0]=\{x\}$. Then, the function

$$
h(u, v)= \begin{cases}g(u, 0), & u \in F_{k}, v=1 / k \\ 2-g(u, 0), & u \in F_{k}, v=-1 / k \\ 1, & \text { otherwise }\end{cases}
$$

is in $\mathcal{H}_{2}, 0 \leq h \leq 2$,

$$
[h=0]=\{(x, 1 / k)\} \quad \text { and } \quad[h=2]=\{(x,-1 / k)\} .
$$

Thus, both the points $(x, 1 / k)$ and $(x,-1 / k)$ are in $\mathrm{Ch}_{\mathcal{H}_{2}} K_{2}$.

To check that $\mathcal{H}_{2}$ is simplicial, we show the following.

Claim. Let

$$
H=F \cup \bigcup_{k=1}^{n}\left(F_{k} \times\{1 / k\}\right) \cup\left(F_{k} \times\{-1 / k\}\right),
$$

where $F \subset \mathrm{Ch}_{\mathcal{H}_{1}} K_{1} \backslash \bigcup_{k} F_{k}$ is compact and $n \in \mathbb{N}$. Then, for any continuous function $f$ on $H$ there exists $h \in \mathcal{H}_{2}$ such that $\|h\|=\|f\|$ and $h=f$ on $H$.

Let $H$ be as in the premise and $f$ be a continuous function on $H$. Then $\widehat{H}=$ $F \cup \bigcup_{k=1}^{n} F_{k}$ is a compact subset of $\mathrm{Ch}_{\mathcal{H}_{1}} K_{1}$ and

$$
\widehat{f}(x, y)= \begin{cases}f(x, y), & (x, y) \in F \\ \frac{1}{2}(f(x, 1 / k)+f(x,-1 / k)), & x \in F_{k}, y=0\end{cases}
$$

is a continuous function on $\widehat{H}$. Since $\mathcal{H}_{1}$ is simplicial and $\mathcal{A}_{c}\left(\mathcal{H}_{1}\right)=\mathcal{H}_{1}$, by Theorem 2.6) (a) there exists a function $\widehat{h} \in \mathcal{H}_{1}$ such that $\|\widehat{h}\|=\|\widehat{f}\|$ and $\widehat{h}=\widehat{f}$ on $\widehat{H}$. Then, the function

$$
h= \begin{cases}\widehat{h}, & \text { on } K_{1}, \\ f, & \text { on } \bigcup_{k=1}^{n}\left(F_{k} \times\{1 / k\}\right) \cup\left(F_{k} \times\{-1 / k\}\right), \\ \widehat{h} \circ p, & \text { otherwise }\end{cases}
$$

is in $\mathcal{H}_{2}, h=f$ on $H$ and $\|h\|=\|f\|$. This concludes the proof of the claim. 
Assume now that $\mu$ and $\nu$ are $\mathcal{H}_{2}$-representing maximal measures for a point $x \in K_{2}, f \in \mathcal{C}\left(K_{2}\right)$ and $\varepsilon>0$. Since maximal measures are supported by $\mathrm{Ch}_{\mathcal{H}_{2}} K_{2}$, there exist $n \in \mathbb{N}$ and a compact set $F \subset \mathrm{Ch}_{\mathcal{H}_{1}} K_{1} \backslash \bigcup_{k} F_{k}$ such that the set

$$
H=F \cup \bigcup_{k=1}^{n}\left(F_{k} \times\{1 / k\}\right) \cup\left(F_{k} \times\{-1 / k\}\right)
$$

satisfies

$$
(\mu+\nu)\left(K_{2} \backslash H\right)<\varepsilon .
$$

By the claim above, we can find a function $h \in \mathcal{H}_{2}$ such that $\|h\|=\|f\|$ and $h=f$ on $H$. Then

$$
|\mu(f)-\nu(f)| \leq|\mu(f)-\mu(h)|+|\nu(f)-\nu(h)| \leq 4 \varepsilon\|f\| .
$$

Hence, $\mu(f)=\nu(f)$ for any $f \in \mathcal{C}\left(K_{2}\right)$ and $\mu=\nu$. Thus $\mathcal{H}_{2}$ is simplicial.

To verify (f), let $f$ be as in the premise. Given a point $x \in K_{1}$ and an $\mathcal{H}_{1}-$ maximal measure $\mu \in \mathcal{M}_{x}\left(\mathcal{H}_{1}\right)$, we consider the measure $\nu$ defined as

$$
\begin{aligned}
\nu(g)= & \int_{K_{1} \backslash \cup_{k} F_{k}} g(t, 0) d \mu(t) \\
& +\sum_{k=1}^{\infty} \int_{F_{k}} \frac{1}{2}(g(t, 1 / k)+g(t,-1 / k)) d \mu(t), \quad g \in \mathcal{C}\left(K_{2}\right) .
\end{aligned}
$$

Then, $\nu \in \mathcal{M}_{x}\left(\mathcal{H}_{2}\right)$ and $\nu$ is $\mathcal{H}_{2}-$ maximal as it is supported by $\mathrm{Ch}_{\mathcal{H}_{2}} K_{2}$. By (e), $\nu$ is the unique $\mathcal{H}_{2}$-maximal measure $\mathcal{H}_{2}-$ representing $x$. By the assumption,

$$
\nu(f)=\int_{K_{1}} f(t, 0) d \mu(t)=f(x, 0) .
$$

This along with (d) implies that $\nu(f)=f(x)$ for every $x \in K_{2}$ and $\mathcal{H}_{2}$-maximal measure $\nu \in \mathcal{M}_{x}\left(\mathcal{H}_{2}\right)$.

According to Theorem 2.6(b3), $f$ is $\mathcal{H}_{2}$-affine. This concludes the proof.

5.2. Inductive construction. Let $\left\{F_{s}: s \in \mathbb{N}^{<\mathbb{N}}\right\}$ be the family of perfect sets in $[0,1]$ provided by Lemma 3.3. If $x$ is a point of $\mathbb{R}^{n+1}$, we write $x$ in coordinates as $(x(0), x(1), \ldots, x(n))$.

For every $n \geq 0$, we construct by induction a simplicial function space $\mathcal{H}_{n}$ on a metrizable compact space $K_{n} \subset \mathbb{R}^{n+1}$, a countable family $\mathcal{F}_{n}=\left\{F_{n}(k): k \in \mathbb{N}\right\}$ of pairwise disjoint compact sets in $\mathrm{Ch}_{\mathcal{H}_{n}} K_{n}$ and a continuous surjection $p_{n+1}$ : $K_{n+1} \rightarrow K_{n}$ as follows.

In the first step, let $K_{0}=[0,1], \mathcal{H}_{0}=\mathcal{C}([0,1])$ and $\mathcal{F}_{0}=\left\{F_{s}:|s|=1\right\}$.

Assume that the objects have been defined for each $k=0, \ldots, n$. We use Lemma 5.1 on $\mathcal{H}_{n}, K_{n}$ and $\mathcal{F}_{n}$ to obtain

$$
K_{n+1} \subset K_{n} \times \mathbb{R} \subset \mathbb{R}^{n+2} \text { and } \mathcal{H}_{n+1} \text {. }
$$

Let $p_{n+1}: K_{n+1} \rightarrow K_{n}$ be the natural projection.

Given that the family $\mathcal{F}_{n}$ is enumerated as $\mathcal{F}_{n}=\{F(k): k \in \mathbb{N}\}$, for each $k \in \mathbb{N}$ and a sequence $s \in \mathbb{N}^{n+2}$ of length $n+2$, we consider the following couple of sets:

$$
\begin{aligned}
F(s, k,+)=\{x & =(x(0), \ldots, x(n+1)) \in K_{n+1}: \\
p_{n+1}(x) & \left.=(x(0), \ldots, x(n)) \in F(k), x(0) \in F_{s}, x(n+1)=1 / k\right\}, \\
F(s, k,-)=\{x & =(x(0), \ldots, x(n+1)) \in K_{n+1}: \\
p_{n+1}(x) & \left.=(x(0), \ldots, x(n)) \in F(k), x(0) \in F_{s}, x(n+1)=-1 / k\right\} .
\end{aligned}
$$


We remark that the set $F(s, k,+)$ (respectively $F(s, k,-)$ ) is a closed subset of $F(k) \times\{1 / k\} \subset K_{n+1}$ (respectively $\left.F(k) \times\{-1 / k\}\right)$ and hence a closed subset of $K_{n+1} \backslash K_{n} \subset \mathrm{Ch}_{\mathcal{H}_{n+1}} K_{n+1}$.

We set

$$
\mathcal{F}_{n+1}=\left\{F(s, k,+), F(s, k,-): s \in \mathbb{N}^{n+2}, k \in \mathbb{N}\right\} .
$$

This finishes the inductive step.

5.3. Definition of a function space. We have just obtained a sequence $\left\{\mathcal{H}_{n}\right\}$ of simplicial spaces on compact metrizable spaces $\left\{K_{n}\right\}$ together with surjective continuous mappings $p_{n}$, in short,

$$
K_{0} \stackrel{p_{1}}{\longleftarrow} K_{1} \stackrel{p_{2}}{\longleftarrow} K_{2} \leftarrow \cdots \text {. }
$$

Let $K=\lim _{\leftarrow} K_{n}$ be the limit of the inverse system (9) (see [10, Chapter 2.5]), i.e.,

$$
K=\left\{x=\left\{x_{n}\right\} \in \prod_{n=0}^{\infty} K_{n}: p_{n+1}\left(x_{n+1}\right)=x_{n}, n \geq 0\right\}
$$

with the product topology. Hence a typical element $x \in K$ is of the form $x=$ $\left(x_{0}, x_{1}, x_{2}, \ldots\right)$, where

$$
\begin{aligned}
& x_{0}=\left(x_{0}(0)\right) \in K_{0} \subset \mathbb{R}, \\
& x_{1}=\left(x_{1}(0), x_{1}(1)\right) \in K_{1} \subset \mathbb{R}^{2}, \\
& x_{2}=\left(x_{2}(0), x_{2}(1), x_{2}(2)\right) \in K_{2} \subset \mathbb{R}^{3},
\end{aligned}
$$

and

$$
\begin{aligned}
& x_{0}(0)=x_{1}(0)=x_{2}(0)=x_{3}(0)=\ldots, \\
& x_{1}(1)=x_{2}(1)=x_{3}(1)=\ldots, \\
& x_{2}(2)=x_{3}(2)=\ldots,
\end{aligned}
$$

Then $K$ is a metrizable compact space and we can consider each compact space $K_{n}$ homeomorphically embedded in $K$ via the mapping

$$
\begin{aligned}
e_{n}: K_{n} & \rightarrow K, \\
x & \mapsto\left(\left(p_{1} \circ \cdots \circ p_{n}\right)(x), \ldots,\left(p_{n-1} \circ p_{n}\right)(x), p_{n}(x), \stackrel{n-\text { th }}{x}, x, \ldots\right) .
\end{aligned}
$$

Conversely, we can define retractions of $K$ onto $K_{n}$ as

$$
\begin{aligned}
r_{n}: & K \rightarrow K_{n}, \\
& \left\{x_{n}\right\} \mapsto\left(x_{0}, \ldots, x_{n-1}, x_{n}, x_{n}, x_{n} \ldots\right) .
\end{aligned}
$$

Using these mappings we can regard each function space $\mathcal{H}_{n}$ as a subspace of $\mathcal{C}(K)$; more precisely, we use the mapping

$$
h \mapsto h \circ r_{n}, \quad h \in \mathcal{H}_{n} .
$$

In the sequel we will use these identifications without referring to (10).

We fix $n \geq 0$. For $x=\left(x_{0}, x_{1}, x_{2}, \ldots\right) \in K$, we write $x_{n} \in K_{n} \subset \mathbb{R}^{n+1}$ in coordinates as

$$
x_{n}=\left(x_{n}(0), x_{n}(1), \ldots, x_{n}(n)\right) .
$$


We define a "coordinate" function $c_{n}: K \rightarrow \mathbb{R}$ as

$$
c_{n}(x)=x_{n}(n), \quad x \in K .
$$

We define a function space $\mathcal{H}$ on $K$ as

$$
\mathcal{H}=\overline{\bigcup_{n=1}^{\infty} \mathcal{H}_{n}} .
$$

\section{Properties of $\mathcal{H}$}

Lemma 6.1. Let $\mathcal{H}$ be the space defined above. Then

(a) the space $\mathcal{H}$ is a simplicial function space;

(b) $\mathcal{H}=\mathcal{A}_{c}(\mathcal{H})$;

(c) $K \backslash \mathrm{Ch}_{\mathcal{H}} K=\bigcup_{n>0} \cup \mathcal{F}_{n}$;

(d) if $n \geq 0$ and $f \in \mathcal{A}\left(\mathcal{H}_{n}\right)$, then $f \circ r_{n} \in \mathcal{A}(\mathcal{H})$.

Proof. To start the proof of (a) we remark that $\mathcal{H}$ contains constant functions. To show that it separates points, let $x, y \in K$ be a couple of distinct points. Let $x_{n} \neq y_{n}$ for some $n \geq 0$ and let $h \in \mathcal{H}_{n}$ satisfy $h\left(x_{n}\right) \neq h\left(y_{n}\right)$. Taking into consideration the embedding of $\mathcal{H}_{n}$ in $\mathcal{H}, h$ is the required function distinguishing $x$ and $y$.

For the proof of simpliciality of $\mathcal{H}$ it is enough to verify the weak Riesz interpolation property. As all spaces $\mathcal{H}_{n}$ possess this property and $\mathcal{H}$ is the closure of their increasing union, $\mathcal{H}$ is simplicial.

Also by Theorem 2.6(a), $\mathcal{H}=\mathcal{A}_{c}(\mathcal{H})$.

To verify (c), let $x \in \bigcup \mathcal{F}_{n}$ for some $n \geq 0$. Let $a, b$ be the unique points of $K_{n+1} \backslash K_{n}$ such that $x=p_{n+1}(a)=p_{n+1}(b)$. It follows from the construction that the measure $\frac{1}{2}\left(\varepsilon_{a}+\varepsilon_{b}\right)$ is an $\mathcal{H}$-representing measure for $x$. Thus $x \in K \backslash \mathrm{Ch}_{\mathcal{H}} K$.

On the other hand, if $x \in K_{n} \backslash \bigcup_{j=0}^{n} \cup \mathcal{F}_{j}, n \geq 0$, then $x \in \mathrm{Ch}_{\mathcal{H}_{n}} K_{n}$. By Theorem 6.1](b), there exists an $\mathcal{H}_{n}$-exposing function $h$ for $x$. Then $h \circ r_{n}$ is an $\mathcal{H}$-exposing function for $x$ and $x \in \mathrm{Ch}_{\mathcal{H}} K$.

If $x=\left(x_{0}, x_{1}, \ldots\right) \in K \backslash \bigcup_{n} K_{n}$, then $r_{n}(x) \in \mathrm{Ch}_{\mathcal{H}_{n}} K_{n}$ for each $n \geq 0$. For each $n \geq 0$, let $h_{n}$ be an $\mathcal{H}_{n}$-exposing function for $r_{n}(x)$ with $0 \leq h_{n} \leq 1$. Then $h=\sum_{n>0} \frac{1}{2^{n}}\left(h_{n} \circ r_{n}\right)$ is an $\mathcal{H}$-exposing function for $x$. Thus $x \in \mathrm{Ch}_{\mathcal{H}} K$.

For the proof of (d), let $f$ be a function in some $\mathcal{A}\left(\mathcal{H}_{n}\right)$. Given a point $x \in K$ and a measure $\mu \in \mathcal{M}_{x}(\mathcal{H})$, we notice that $r_{n} \mu \in \mathcal{M}_{r_{n}(x)}\left(\mathcal{H}_{n}\right)$. Indeed, given a function $h \in \mathcal{H}_{n}$, then

$$
\left(r_{n} \mu\right)(h)=\mu\left(h \circ r_{n}\right)=\left(h \circ r_{n}\right)(x) .
$$

Thus,

$$
\mu\left(f \circ r_{n}\right)=\left(r_{n} \mu\right)(f)=f\left(r_{n}(x)\right),
$$

and $f \circ r_{n}$ is $\mathcal{H}$-affine. This concludes the proof.

6.1. Cantor sets. Let $A$ be the subset of $[0,1]$ defined as $A=\bigcap_{n} \bigcup_{|s|=n} F_{s}$. For any point $a$ of $A$ we define a set $C_{a} \in K \backslash \bigcup_{n} K_{n}$ as

$$
C_{a}=\left\{x=\left(x_{0}, x_{1}, x_{2}, \ldots\right) \in K \backslash \bigcup_{n=0}^{\infty} K_{n}: x_{0}=a\right\} .
$$


We define a mapping

$$
\begin{aligned}
\varphi_{a}: & \{0,1\}^{\mathbb{N}} \rightarrow C_{a}, \\
& \left(\tau_{1}, \tau_{2}, \ldots,\right) \mapsto x=\left(a, x_{1}, x_{2}, \ldots\right),
\end{aligned}
$$

so that

$$
c_{n}(x)=x_{n}(n)\left\{\begin{array}{ll}
>0, & \tau_{n}=1, \\
<0, & \tau_{n}=0,
\end{array} \quad n \in \mathbb{N} .\right.
$$

(Here $c_{n}: K \rightarrow \mathbb{R}$ is the function from (11).)

For any $n \in \mathbb{N}, a \in \bigcup\left\{F_{s}:|s|=n\right\}$ and $t \in\{0,1\}^{n}$ we define a point

$$
x_{a, t}=\left(x_{a, t}(0), x_{a, t}(1), \ldots, x_{a, t}(n)\right) \in K_{n} \subset \mathbb{R}^{n+1}
$$

by setting

$$
x_{a, t}(0)=a \quad \text { and } \quad x_{a, t}(i)\left\{\begin{array}{ll}
>0, & t_{i}=1, \\
<0, & t_{i}=0,
\end{array} \quad i=1, \ldots, n .\right.
$$

Further, let $f$ be a function on $K$ and $t \in\{0,1\}^{n}$. We define a function

$$
\begin{aligned}
f^{t}: & \bigcup\left\{F_{s}:|s|=n\right\} \rightarrow \mathbb{R}, \\
& f^{t}(a)=f\left(x_{a, t}\right), \quad a \in F_{s},|s|=n .
\end{aligned}
$$

Lemma 6.2. Let $n \in \mathbb{N}, a \in \bigcup\left\{F_{s}:|s|=n\right\}$ and $t \in\{0,1\}^{n}$. Then the point $x_{a, t}$ is unique and well defined. Moreover, if $a \in \bigcup\left\{F_{s}:|s|=n+1\right\}$, then $x_{a, t} \in \bigcup \mathcal{F}_{n}$.

Proof. We prove the assertion by induction.

If $n=1, a \in F_{s}$ for some $s$ of length 1 and $t \in\{0,1\}$, let $k \in \mathbb{N}$ be the index of the set $F_{s}$ in the enumeration $\left\{F_{s}:|s|=1\right\}=\{F(k): k \in \mathbb{N}\}$ (see Inductive Construction 5.2 and Lemma 5.1). Then

$$
x_{a, t}= \begin{cases}\left(a, \frac{1}{k}\right), & \text { if } t=1, \\ \left(a,-\frac{1}{k}\right), & \text { if } t=0 .\end{cases}
$$

If $a \in F_{s^{\prime}}$ for some $s^{\prime} \in \mathbb{N}^{2}$, then $x_{a, t}$ is contained either in $F\left(s^{\prime}, k,+\right)$ or in $F\left(s^{\prime}, k,-\right)$ (see (8) ). Hence $x_{a, t} \in \bigcup \mathcal{F}_{1}$ as needed.

Assume that $n \in \mathbb{N}$ and that the verification has been done for all $k \leq n$. We select any $a \in \bigcup\left\{F_{s}:|s|=n+1\right\}, t \in\{0,1\}^{n}$ and $i \in\{0,1\}$ and check that the point $x_{a, t^{\wedge} i}$ is well defined. According to the inductive assumption, the point $x_{a, t}$ is contained in $\bigcup \mathcal{F}_{n}$. Given $\mathcal{F}_{n}=\{F(k): k \in \mathbb{N}\}$ as the enumeration of the family $\mathcal{F}_{n}$, let $k \in \mathbb{N}$ be such that $x_{a, t} \in F(k)$. By Inductive Construction 5.2 ,

$$
x_{a, t^{\wedge} i}= \begin{cases}\left(a, x_{a, t}(1), \ldots, x_{a, t}(n), \frac{1}{k}\right), & \text { if } i=1, \\ \left(a, x_{a, t}(1), \ldots, x_{a, t}(n),-\frac{1}{k}\right), & \text { if } i=0 .\end{cases}
$$

If $a \in F_{s^{\prime}}$ for some $s^{\prime} \in \mathbb{N}^{n+2}$, then as above we get that $x_{a, t^{\wedge} i}$ is contained either in $F\left(s^{\prime}, k,+\right)$ or in $F\left(s^{\prime}, k,-\right)$, depending on the fact whether $i=1$ or 0 . Hence $x_{a, t^{\wedge} i} \in \bigcup \mathcal{F}_{n+1}$, which finishes the proof.

Lemma 6.3. For any $a \in A$, the mapping $\varphi_{a}$ is a well-defined homeomorphism of $\{0,1\}^{\mathbb{N}}$ onto $C_{a}$. 
Proof. Let $a \in \bigcap_{n} \bigcup_{|s|=n} F_{s}$ be given and let $C_{a}$ and $\varphi_{a}$ be defined as in Lemma 6.1. It follows from the construction that the set $C_{a}$ as well as the mapping $\varphi_{a}$ is well defined. Moreover, it is surjective because any point $\left(a, x_{1}, x_{2}, \ldots\right) \in C_{a}$ is uniquely determined by signs of $x_{1}(1), x_{2}(2), x_{3}(3), \ldots$. From this observation it also follows that $\varphi_{a}$ is injective.

Let $\tau \in\{0,1\}^{\mathbb{N}}$ be given. We claim that $x_{a,\left.\tau\right|_{n}} \rightarrow \varphi_{a}(\tau)$ in $K$ as $n \rightarrow \infty$. If $\varphi_{a}(\tau)=\left(a, x_{1}, x_{2}, \ldots\right)$, it follows from the definitions in Lemma 6.1 that

$$
x_{a,\left.\tau\right|_{n}}=x_{n}, \quad n \in \mathbb{N} .
$$

If we take into account the identification

$$
\begin{aligned}
x_{a,\left.\tau\right|_{n}} & =\left(\left(p_{1} \circ \cdots \circ p_{n}\right)\left(x_{a,\left.\tau\right|_{n}}\right), \ldots, p_{n}\left(x_{a,\left.\tau\right|_{n}}\right), x_{a,\left.\tau\right|_{n}}^{n-\text { th }}, x_{a,\left.\tau\right|_{n}}, \ldots\right) \\
& =\left(a, x_{1}, \ldots, x_{n-1}, x_{n}^{n-\text { th }}, x_{n}, \ldots\right), \quad n \in \mathbb{N},
\end{aligned}
$$

from the definition of a function space in 5.3 , we get the desired conclusion since $K \subset \prod_{n} K_{n}$ is endowed with the product topology.

Further, let $\tau^{j} \rightarrow \tau$ in $\{0,1\}^{\mathbb{N}}$ as $j \rightarrow \infty$. Then $\varphi_{a}\left(\tau^{j}\right)=\left(a, x_{1}^{j}, x_{2}^{j}, \ldots\right)$, where $x_{n}^{j}=x_{a,\left.\tau^{j}\right|_{n}}, n, j \in \mathbb{N}$. For fixed $n \in \mathbb{N},\left.\tau^{j}\right|_{n}=\left.\tau\right|_{n}$ for sufficiently large indices $j$. Hence

$$
\varphi_{a}\left(\tau^{j}\right)=\left(a, x_{a,\left.\tau^{j}\right|_{1}}, x_{a,\left.\tau^{j}\right|_{2}}, \ldots\right) \underset{j \rightarrow \infty}{\longrightarrow}\left(a, x_{a,\left.\tau\right|_{1}}, x_{a,\left.\tau\right|_{2}}, \ldots\right)=\varphi_{a}(\tau)
$$

and $\varphi_{a}$ is a continuous mapping. Since it is injective, it is a homeomorphism.

Lemma 6.4. For every $a \in A$ and $t \in\{0,1\}^{m}$, it follows that $\delta_{x_{a, t}}=\varphi_{a} \mu_{t}$.

Proof. Let $a \in A$ and $t \in\{0,1\}^{m}$ be as in the premise. Since $\varphi_{a} \mu_{t}$ is supported by $K \backslash \bigcup_{n} K_{n}, \varphi_{a} \mu_{t}$ is an $\mathcal{H}$-maximal measure. As $\mathcal{H}$ is simplicial, to finish the proof it is enough to show that $\varphi_{a} \mu_{t}$ is an $\mathcal{H}$-representing measure for $x_{a, t}$.

To this end, we pick a function $h$ in some $\mathcal{H}_{n}$. We notice that

$$
h(x)=h\left(x_{a, s}\right) \text { if } r_{n}(x)=x_{a, s}, \quad x \in K, s \in\{0,1\}^{n} .
$$

Thus, $\varphi_{a} \mu_{t}(h)=h\left(x_{a, t}\right)$ if $n \leq m$.

If $n>m$, then by (12) and Construction 5.2,

$$
\begin{aligned}
\varphi_{a} \mu_{t}(h) & =\varphi_{a}\left(\sum_{\substack{\left|t^{\prime}\right|=n \\
t \subset t^{\prime}}} \frac{1}{2^{n-m}} \mu_{t^{\prime}}\right)(h)=\sum_{\substack{\left|t^{\prime}\right|=n \\
t \subset t^{\prime}}} \frac{1}{2^{n-m}}\left(\varphi_{a} \mu_{t^{\prime}}\right)(h) \\
& =\sum_{\substack{\left|t^{\prime}\right|=n \\
t \subset t^{\prime}}} \frac{1}{2^{n-m}} h\left(x_{a, t^{\prime}}\right)=h\left(x_{a, t}\right) .
\end{aligned}
$$

Since $\bigcup_{n} \mathcal{H}_{n}$ is dense in $\mathcal{H}, \varphi_{a} \mu_{t}$ is an $\mathcal{H}$-representing measure for $x_{a, t}$. This concludes the proof.

6.2. Definition of a Baire-two function. We define a function $f$ on $K$ as

$$
f(x)= \begin{cases}\operatorname{sgn} c_{n}(x) & \text { if } x \in K_{n} \backslash\left(K_{n-1} \cup \cup \mathcal{F}_{n}\right), n \in \mathbb{N}, \\ 0 & \text { otherwise } .\end{cases}
$$

Lemma 6.5. The function $f$ is $\mathcal{H}$-affine and Baire-two. 
Proof. First, we show inductively that $f$ is Baire-two on $K_{n-1}$ for each $n \in \mathbb{N}$. Indeed, $f=0$ on $K_{0}$ and assuming that the assertion has been established for $n \in \mathbb{N}$, we notice that $K_{n} \backslash K_{n-1}$ is an open set in $K_{n-1}$ and

$$
\left.f\right|_{K_{n} \backslash K_{n_{1}}}=\chi_{G_{1}}-\chi_{G_{2}},
$$

where

$$
\begin{aligned}
& G_{1}=\left\{x \in K_{n}: x_{n}(n)>0\right\} \cap\left(K_{n} \backslash\left(K_{n-1} \cup \mathcal{F}_{n}\right)\right) \text { and } \\
& G_{2}=\left\{x \in K_{n}: x_{n}(n)<0\right\} \cap\left(K_{n} \backslash\left(K_{n-1} \cup \mathcal{F}_{n}\right)\right) .
\end{aligned}
$$

Since $\bigcup \mathcal{F}_{n}$ is an $F_{\sigma}$-set and both $\left\{x \in K_{n}: x_{n}(n)>0\right\}$ and $\left\{x \in K_{n}: x_{n}(n)>0\right\}$ are open in $K_{n}$, the sets $G_{1}$ and $G_{2}$ are $G_{\delta}$-subsets of $K_{n} \backslash K_{n-1}$. From this the assertion follows.

Hence $f$ is a Baire-two function on each $K_{n}$. Further, $K_{n} \in \boldsymbol{\Sigma}_{1}^{0}(K), n \in \mathbb{N}$, and $\bigcup_{n} K_{n} \in \Delta_{3}^{0}(K)$ and $f=0$ on $K \backslash \bigcup_{n} K_{n}$. Thus by setting $A_{n}=K_{n}$ in Lemma 3.4 we get that $f$ is Baire-two on $K$.

To check that $f$ is $\mathcal{H}$-affine, we consider the restrictions $f_{n}=\left.f\right|_{K_{n}}, n \geq 0$. By an inductive argument we get from Lemma 5.1 that $f_{n} \in \mathcal{A}\left(\mathcal{H}_{n}\right), n \geq 0$. By Lemma 6.1(d), $f_{n} \circ r_{n} \in \mathcal{A}(\mathcal{H})$. Since $f_{n} \circ r_{n} \rightarrow f, f$ is $\mathcal{H}$-affine.

Lemma 6.6. The function $f$ defined in Lemma 6.2 is not a pointwise limit of a bounded sequence of $\mathcal{H}$-affine Baire-one functions.

Proof. Assume that $\left\{f_{n}\right\}$ is a bounded sequence of $\mathcal{H}$-affine Baire-one functions pointwise converging to $f$. We choose a number $\varepsilon^{\prime} \in(0,1)$.

We will construct by induction finite sequences $s_{n} \in \mathbb{N}<\mathbb{N}$, indices $k_{n} \in \mathbb{N}$ and nonempty relatively open subsets $V_{n} \subset F_{s_{n}}, n \geq 1$, such that

(i) $s_{1}=1$;

(ii) $\left|s_{n}\right|=n, s_{n} \subset s_{n+1}$;

(iii) $k_{n}<k_{n+1}$

(iv) $\left|f_{k_{n}}^{t}(a)-(-1)^{t_{n}+1}\right|<\varepsilon^{\prime}$ for each $a \in V_{n}$ and $t \in\{0,1\}^{n}$;

(v) $F_{s_{n+1}} \subset V_{n}$.

To start the construction, we set $s_{1}=1$ and use Lemma 3.5 for $\varepsilon^{\prime}$, the compact space $F_{s_{1}}$, sequences $\left\{f_{k}^{0}\right\},\left\{f_{k}^{1}\right\}$ (here 0 or 1 stands for the sequence of length 1 consisting of the digit 0 or 1 , respectively), dense $G_{\delta}$-subsets of $F_{s_{1}}$,

$$
G_{0}=G_{1}=F_{s_{1}} \backslash \bigcup_{j=1}^{\infty} F_{s_{1} \wedge j}
$$

and numbers $a_{0}=-1, a_{1}=1$.

We get an index $k_{1} \geq 1$ and a relatively open subset $V_{1}$ of $F_{s_{1}}$ such that

$$
\left|f_{k_{1}}^{i}(a)-(-1)^{i+1}\right|<\varepsilon^{\prime}, \quad a \in V_{1}, i=0,1 .
$$

This concludes the first step.

Assume now that the construction has been completed up to the $n$-th stage. We find $j \in \mathbb{N}$ such that $F_{s_{n} \wedge j} \subset V_{n}$ and set $s_{n+1}=s_{n} \wedge j$. We use Lemma 3.5 for $\varepsilon^{\prime}$, the compact space $F_{s_{n+1}}$, dense $G_{\delta}$-subsets of $F_{s_{n+1}}$,

$$
G_{t}=F_{s_{n+1}} \backslash \bigcup_{j=1}^{\infty} F_{s_{n+1} \wedge}, \quad t \in\{0,1\}^{n+1},
$$

sequences $\left\{\left\{f_{k}^{t}\right\}: t \in\{0,1\}^{n+1}\right\}$ and numbers $\left\{(-1)^{t_{n+1}+1}: t \in\{0,1\}^{n+1}\right\}$. 
We get an index $k_{n+1}>k_{n}$ and a relatively open subset $V_{n+1}$ of $F_{s_{n+1}}$ so that

$$
\left|f_{k_{n+1}}^{t}(a)-(-1)^{t_{n+1}+1}\right|<\varepsilon^{\prime}, \quad a \in V_{n+1}, t \in\{0,1\}^{n+1} .
$$

This finishes the construction.

Let $\sigma \in \mathbb{N}^{\mathbb{N}}$ satisfy $\left.\sigma\right|_{n}=s_{n}, n \in \mathbb{N}$, and let $a \in \bigcap_{n} F_{\left.\sigma\right|_{n}}$. Let $C_{a}$ and $\varphi_{a}$ be defined as in Lemma 6.1. We define functions $g_{n}:\{0,1\}^{\mathbb{N}} \rightarrow \mathbb{R}, n \in \mathbb{N}$, as

$$
g_{n}(\sigma)=f_{k_{n}}\left(\varphi_{a}(\sigma)\right), \quad \sigma \in\{0,1\}^{\mathbb{N}}
$$

Then $g_{n} \rightarrow 0$ since $f_{k_{n}} \rightarrow 0$ on $K \backslash \bigcup_{n} K_{n}$.

Let $t \in\{0,1\}^{<\mathbb{N}}$ be fixed. According to Lemma 6.4 $\delta_{x_{a, t}}=\varphi_{a} \mu_{t}$. Hence

$$
f_{k_{n}}^{t}(a)=f_{k_{n}}\left(x_{a, t}\right)=\delta_{x_{a, t}}\left(f_{k_{n}}\right)=\varphi_{a} \mu_{t}\left(f_{k_{n}}\right)=\mu_{t}\left(g_{n}\right) .
$$

Using condition (iv) we get that the sequence $\left\{g_{n}\right\}$ satisfies the assumptions of Lemma 3.6

But this is impossible as $g_{n} \rightarrow 0$. This contradiction finishes the proof.

\section{Proofs of the main Results}

Proof of Theorem 1.1. Let $\mathcal{H}$ be the simplicial function space on the metrizable space $K$ constructed in Section 5 and let $f$ be the function from Lemma 6.2. By Theorem 2.6(a), the state space $X=\mathbf{S}(\mathcal{H})$ is a simplex. According to Theorem 2.6 (c), Lemma 6.5] and Theorem 2.5(d), the function $\widehat{f}=\left.I f\right|_{X}$ is contained in $\mathfrak{A}_{\mathrm{bf}}(\mathrm{X}) \cap \mathcal{B}_{2}(\mathrm{X})$.

We claim that $\widehat{f} \notin \mathfrak{A}_{2}(X)$. Assuming a contradiction, there exists a bounded sequence $\left\{\widehat{f}_{n}\right\} \subset \mathfrak{A}_{1}(X)$ pointwise converging to $\widehat{f}$. Then $f_{n}=I^{-1} \widehat{f}_{n}, n \in \mathbb{N}$, are contained in $\mathcal{H}_{1}$ and $f_{n} \rightarrow f$. But this is impossible by Lemma 6.6. This concludes the proof.

Remark 7.1. For the sake of completeness, we present the proof of the fact that the space $\mathfrak{A}(X)$ on the simplex $X$ constructed in Theorem 1.1 is not complemented in any $\mathcal{C}(L)$-space.

Indeed, if it were true, then the function space $\mathcal{H}$ constructed in $\$ 5.3$ would be complemented in a $\mathcal{C}(L)$-space as well. According to Proposition 4.3 , $\mathcal{H}_{2}=$ $\mathcal{B}_{2}(K) \cap \mathcal{H}^{\perp \perp}$, a contradiction with Lemma 6.6.

Proof of Theorem 1.2. If $\mathcal{H}$ is the function space from $\$ 5.3$, then $\mathcal{H}$ is an $L_{1}$-predual (it is isometric with $\mathfrak{A}(\mathbf{S}(\mathcal{H}))$ ) and thus an $\mathcal{L}_{\infty, 1+\varepsilon}$-space for every $\varepsilon>0$ (see [13, page 59]).

By Theorem 2.5(d), (f) and Lemma 6.6

$$
\mathcal{H}_{2}^{* *}=I\left(\mathcal{H}_{2}\right) \neq I\left(\mathcal{B}_{2}(K) \cap \mathcal{H}^{\perp \perp}\right)=\mathcal{H}_{\mathcal{B}_{2}}^{* *} .
$$

Proof of Example 1.3, Let $X$ be the simplex $X_{2}$ constructed in [4, Theorem 1.1]. Then, $X$ is metrizable, ext $X$ is countable and $Y=\mathfrak{A}(X)$ is not complemented in any $\mathcal{C}(K)$-space. By a folklore result (see e.g. [22, Théorème 37$]), \mathcal{B}_{\alpha}(X) \cap \mathfrak{A}_{\mathrm{bf}}(\mathrm{X})=$ $\mathfrak{A}_{\alpha}(\mathrm{X})$ for each $\alpha \in\left[0, \omega_{1}\right)$. Using Theorem 2.5, we get for each $\alpha \in\left[0, \omega_{1}\right)$,

$$
Y_{\alpha}^{* *}=I\left(\mathfrak{A}_{\alpha}(X)\right)=I\left(\mathcal{B}_{\alpha}(X) \cap \mathfrak{A}_{\mathrm{bf}}(\mathrm{X})\right)=I\left(\mathcal{B}_{\alpha}(\mathrm{X}) \cap \mathfrak{A}(\mathrm{X})^{\perp \perp}\right)=Y_{\mathcal{B}_{\alpha}}^{* *} .
$$




\section{ACKNOWLEDGEMENT}

The author would like to express his gratitude to his colleagues at the Faculty of Mathematics and Physics of Charles University and at the Faculty of Electrical Engineering of Czech Technical University in Prague who helped him substantially during the work on the paper. In particular, he would like to thank Professors P. Holický, O. Kalenda, J. Lukeš, J. Malý, J. Tišer, L. Zajíček and M. Zelený for fruitful discussions and helpful remarks on the subject. He would also like to thank E.F. Slattery and J. Duda for useful remarks on the final version of the paper and the referee for a meticulous verification of the paper and suggestions leading to a substantial improvement of the paper.

\section{REFERENCES}

[1] E. M. Alfsen, Compact convex sets and boundary integrals, Springer-Verlag, New York and Heidelberg, 1971. MR56:3615

[2] S. A. Argyros, G. Godefroy and H. P. Rosenthal, Descriptive set theory and Banach spaces, Handbook of the geometry of Banach spaces, Vol. II, North-Holland, Amsterdam, 2003, 1007-1069. MR:1999190 (2004g:46002)

[3] L. Asimow and A. J. Ellis, Convexity theory and its applications in functional analysis, Academic Press, 1980. MR623459 (82m:46009)

[4] M. Bačák and J. Spurný, Complementability of spaces of affine continuous functions on simplices, Bull. Belg. Math. Soc. Simon Stevin 15 (2008), no. 3, 465-472. MR2457962 (2009j:46002)

[5] R. Baire, Sur la représentation des fonctions discontinues, Acta. Math., 30 1905, 1-48. MR 1555022

[6] H. Bauer, Simplicial function spaces and simplexes, Expo. Math., 1985, 3, 165-168. MR:87c:46009

[7] Y. Benyamini and J. Lindenstrauss, A predual of $l_{1}$ which is not isomorphic to a $C(K)$ space, Israel J. Math. 13, (1972), 246-254. MR0331013 (48:9348)

[8] M. Capon, Sur les fonctions qui vérifient le calcul barycentrique Proc. London Math. Soc., 32 (1), 1976, 163-180. MR0394148 (52:14952)

[9] G. Choquet, Lectures on analysis I - III, W. A. Benjamin, Inc., New York-Amsterdam, 1969. MR 40:3254

[10] R. Engelking, General topology, Heldermann Verlag, Berlin, 1989. MR.1039321 (91c:54001)

[11] V. P. Fonf, J. Lindenstrauss and R. R. Phelps, Infinite dimensional convexity, Handbook of the geometry of Banach spaces, Vol. I, North-Holland, Amsterdam, 2001, 599-670. MR1863703(2003c:46014)

[12] P. Holický, L. Zajíček and M. Zelený, A remark on a theorem of Solecki, Comment. Math. Univ. Carolin. 46 (1) 2005, 43-54. MR2175858 (2006h:54032)

[13] W. B. Johnson and J. Lindenstrauss, Basic concepts in the geometry of Banach spaces, Handbook of the geometry of Banach spaces, Vol. I, North-Holland, Amsterdam, 2001, 1-84. MR.1863689 (2003f:46013)

[14] A. S. Kechris, Classical descriptive set theory, Springer-Verlag, New York, 1995. MR1321597 (96e:03057)

[15] U. Krause, Der Satz von Choquet als ein abstrakter Spektralsatz und vice versa, Math. Ann., 184 (1970), 275-296. MR 1513280

[16] A. Lazar, Spaces of affine continuous functions on simplexes, Trans. Amer. Math. Soc., 134 (1968), 503-525. MR0233188 (38:1511)

[17] J. Lukeš, J. Malý, I. Netuka, M. Smrčka and J. Spurný, On approximation of affine Baire-one functions, Israel J. Math. 134 (2003), 255-289. MR1972180 (2003m:46056)

[18] J. Lukeš, T. Mocek, M. Smrčka and J. Spurný, Choquet like sets in function spaces, Bull. Sci. Math., 127 (2003), 397-437. MR1991463 (2004f:46038)

[19] N. N. Luzin, Collected works, Part 2, Moscow, 1958. MR0153522 (27:3487)

[20] R. R. Phelps, Lectures on Choquet's theorem. Second edition, Lecture Notes in Mathematics, 1757, Springer-Verlag, Berlin, 2001. MR.1835574(2002k:46001) 
[21] I. Netuka, The Dirichlet problem for harmonic functions, Amer. Math. Monthly, 87 (1980), 621-628. MR82c:31005

[22] M. Rogalski, Opérateurs de Lion, projecteurs boréliens et simplexes analytiques, J. Funct. Anal. 2 (1968), 458-488. MR0236763 (38:5057)

[23] W. Rudin, Functional analysis, International Series in Pure and Applied Mathematics, McGraw-Hill, Inc., New York, 1991. MR.1157815 (92k:46001)

[24] J. Spurný, Representation of abstract affine functions. Real. Anal. Exchange, 2002/2003, 337-354, 28 (2). MR2009758 (2004h:46006)

[25] J. Spurný, The weak Dirichlet problem for Baire functions, Proc. Amer. Math. Soc., 134 (2006), 3153-3157. MR2231897 (2007d:46012)

[26] M. Talagrand, A new type of affine Borel function, Math. Scand., 54 (1984), 183-188. MR757461 (85h:46032)

Faculty of Mathematics and Physics, Charles University, Sokolovská 83, 18675 Praha 8, Czech Republic

E-mail address: spurny@karlin.mff.cuni.cz 